\title{
Exact Solutions for Nonlinear Differential Difference Equations in Mathematical Physics
}

\author{
Khaled A. Gepreel, ${ }^{1,2}$ Taher A. Nofal, ${ }^{2,3}$ and Fawziah M. Alotaibi ${ }^{2}$ \\ ${ }^{1}$ Mathematics Department, Faculty of Science, Zagazig University, Egypt \\ ${ }^{2}$ Mathematics Department, Faculty of Science, Taif University, Saudi Arabia \\ ${ }^{3}$ Mathematics Department, Faculty of Science, Minia University, Egypt \\ Correspondence should be addressed to Khaled A. Gepreel; kagepreel@yahoo.com
}

Received 24 July 2012; Revised 13 October 2012; Accepted 20 November 2012

Academic Editor: Patricia J. Y. Wong

Copyright (C) 2013 Khaled A. Gepreel et al. This is an open access article distributed under the Creative Commons Attribution License, which permits unrestricted use, distribution, and reproduction in any medium, provided the original work is properly cited.

\begin{abstract}
We modified the truncated expansion method to construct the exact solutions for some nonlinear differential difference equations in mathematical physics via the general lattice equation, the discrete nonlinear Schrodinger with a saturable nonlinearity, the quintic discrete nonlinear Schrodinger equation, and the relativistic Toda lattice system. Also, we put a rational solitary wave function method to find the rational solitary wave solutions for some nonlinear differential difference equations. The proposed methods are more effective and powerful to obtain the exact solutions for nonlinear difference differential equations.
\end{abstract}

\section{Introduction}

Nonlinear differential difference equations (NDDEs) play a crucial role in many branches of applied physical sciences such as condensed matter physics, biophysics, atomic chains, molecular crystals, and discretization in solid-state and quantum physics. They also play an important role in numerical simulation of soliton dynamics in high-energy physics because of their rich structures. Therefore, researchers have shown a wide interest in studying NDDEs since the original work of Fermi et al. [1] in the 1950s. Contrary to difference equations that are being fully discretized, NDDEs are semidiscretized, with some (or all) of their space variables being discretized, while time is usually kept continuous. As far as we could verify, little work has been done to search for exact solutions of NDDEs. Hence, it would make sense to do more research on solving NDDEs.

The study of discrete nonlinear system governed by differential difference equations (DDEs) has drawn much attention in recent years particularly from the point of view of complete integrability. There is a vast body of work on nonlinear DDEs, including investigation of integrability criteria, the computation of densities, Backlund transformation, and recursion operator [2-17]. Xie [18] and Zayed et al. [19] have put the rational solitary wave solutions for nonlinear partial differential equations.

In the recent years, there have been a lot of papers devoted to obtain the solitary wave or periodic solutions for a variety of nonlinear differential difference equations by using the symbolic computations. Among these methods Liu [20] used the exponential function rational expansion method to some NDDEs. Zhang et al. [21] have modified the $\left(G^{\prime} / G\right)$ expansion method form solving the nonlinear partial differential equations to solve the nonlinear differential difference equations. Aslan $[22,23]$ has applied the $\left(G^{\prime} / G\right)$ expansion method for solving the discrete nonlinear Schrodinger equations with a saturable nonlinearity, discrete Burgers equation, and the relativistic Toda lattice system. More recently Gepreel et al. [24-26] have used the modified rational Jacobi elliptic functions method to construct some types of Jacobi elliptic solutions of the lattice equation, the discrete nonlinear Schrodinger equation with a saturable nonlinearity, and the quintic discrete nonlinear Schrodinger equation.

In this paper, we use a modified truncated expansion method to construct the exact solutions of the following 
nonlinear difference differential equations in mathematical physics:

(i) the general lattice equation $[21,25,27]$ :

$$
u_{n_{t}}=\left(\alpha+\beta u_{n}+\gamma u_{n}^{2}\right)\left(u_{n+1}-u_{n-1}\right)
$$

(ii) the discrete nonlinear Schrodinger equation with a saturable nonlinearity $[23,25]$ :

$$
i \frac{\partial \psi_{n}}{\partial t}+\left(\psi_{n+1}+\psi_{n-1}-2 \psi_{n}\right)+\frac{\eta\left|\psi_{n}\right|^{2}}{1+\mu\left|\psi_{n}\right|^{2}} \psi_{n}=0
$$

(iii) the quintic discrete nonlinear Schrodinger equation $[26,28]$ :

$$
\begin{aligned}
& i \frac{\partial \psi_{n}}{\partial t}+\alpha\left(\psi_{n+1}-2 \psi_{n}+\psi_{n-1}\right)+\beta\left|\psi_{n}\right|^{2} \psi_{n} \\
& \quad+\gamma\left|\psi_{n}\right|^{2}\left(\psi_{n+1}+\psi_{n-1}\right)+\delta\left|\psi_{n}\right|^{4}\left(\psi_{n+1}+\psi_{n-1}\right)=0
\end{aligned}
$$

(iv) the relativistic Toda lattice system [21]:

$$
\begin{gathered}
u_{n_{t}}-\left(1+\alpha u_{n}\right)\left(v_{n}-v_{n-1}\right)=0, \\
v_{n_{t}}-v_{n}\left(u_{n+1}-u_{n}+\alpha v_{n+1}-\alpha v_{n-1}\right)=0,
\end{gathered}
$$

where $\alpha, \beta, \gamma, \delta, \mu$, and $\eta$ are arbitrary constants. Also, we put a rational solitary wave method to the nonlinear differential difference equations. We use the proposed method to find the rational solitary wave solutions for the nonlinear differential difference equations via the discrete nonlinear Schrodinger equation with a saturable nonlinearity, the quintic discrete nonlinear Schrodinger equation, and the relativistic Toda lattice equation.

\section{Description of the Modified Truncated Expansion Method to Nonlinear DDEs}

In this section, we would like to outline the algorithm for using the modified truncated expansion method to solve the nonlinear DDEs. Consider a given system of $M$ polynomial nonlinear DDEs:

$$
\begin{gathered}
\Delta\left(u_{n+p_{1}}(X), \ldots, u_{n+p_{k}}(X), u_{n+p_{1}}^{\prime}(X), \ldots, u_{n+p_{k}}^{\prime}(X), \ldots,\right. \\
\left.u_{n+p_{1}}^{(r)}(X), \ldots, u_{n+p_{k}}^{(r)}(X)\right)=0
\end{gathered}
$$

where the dependent variable $u$ has $M$ components $u_{i}$, the continuous variable $x$ has $N$ components $x_{j}$, the discrete variable $n$ has $Q$ components $n_{i}$, the $k$ shift vectors $P_{s} \in Z^{\mathrm{Q}}$, and $u^{(r)}$ denotes the collection of mixed derivative terms of order $r$.

The main steps of the algorithm for the modified truncated expansion method to solve NDDEs are outlined as follows.
Step 1. We seek the traveling wave transformation in the following form:

$$
\begin{array}{r}
u_{n+p_{s}}(X)=U\left(\xi_{n+p_{s}}\right), \quad \xi_{n}=\sum_{i=1}^{Q} d_{i} n_{i}+\sum_{j=1}^{m} c_{j} x_{j}+\xi_{0}, \\
s=1,2, \ldots, k
\end{array}
$$

where the coefficients $d_{i}(i=1, \ldots, Q), c_{j}(j=1, \ldots, N)$ and the phase $\xi_{0}$ are constants. The transformations (6) lead to write (5) into the following form:

$$
\begin{aligned}
\Delta( & U_{n+p_{1}}\left(\xi_{n}\right), \ldots, U_{n+p_{k}}\left(\xi_{n}\right), U_{n+p_{1}}^{\prime}\left(\xi_{n}\right), \ldots, \\
& \left.U_{n+p_{k}}^{\prime}\left(\xi_{n}\right), \ldots, U_{n+p_{1}}^{(r)}\left(\xi_{n}\right), \ldots, U_{n+p_{k}}^{(r)}\left(\xi_{n}\right)\right)=0 .
\end{aligned}
$$

Step 2. We suppose the following series expansion as a solution of (7):

$$
U\left(\xi_{n}\right)=\sum_{i=0}^{k} a_{i}\left(\varphi\left(\xi_{n}\right)\right)^{i},
$$

where $a_{i}=(0,1, \ldots, K)$ are arbitrary constants to be determined later, $\varphi\left(\xi_{n}\right)$ has the following form:

$$
\varphi\left(\xi_{n}\right)=\frac{1}{A+e^{\xi_{n}}},
$$

and $A$ is a nonzero arbitrary constant.

Further, using the properties of expansion functions the iterative relations can be written in the following form:

$$
\begin{aligned}
& U_{n+p s}\left(\xi_{n}\right) \\
& =\sum_{i=0}^{K} a_{i}\left(\frac{\varphi\left(\xi_{n}\right) \varphi\left(\sigma_{s}\right)}{A \varphi\left(\xi_{n}\right) \varphi\left(\sigma_{s}\right)(1+A)-A\left[\varphi\left(\sigma_{s}\right)+\varphi\left(\xi_{n}\right)\right]+1}\right)^{i}, \\
& U_{n-p s}\left(\xi_{n}\right) \\
& =\sum_{i=0}^{K} a_{i}\left(\frac{\varphi\left(\xi_{n}\right)-\varphi\left(\sigma_{s}\right) \varphi\left(\xi_{n}\right) A}{A \varphi\left(\xi_{n}\right)+\varphi\left(\sigma_{s}\right)-A \varphi\left(\sigma_{s}\right) \varphi\left(\xi_{n}\right)(1+A)}\right)^{i},
\end{aligned}
$$

where

$$
\xi_{n+p s}=\xi_{n}+\sigma_{s}, \quad \sigma_{s}=p_{s 1} d_{1}+p_{s 2} d_{2}+\cdots+p_{s Q} d_{Q} .
$$

Step 3. Determine the degree $K$ of (8) by balancing the highest order nonlinear term(s) and the highest order derivative of $U\left(\xi_{n}\right)$ in (7).

Step 4. Substituting (8)-(10) and given the value of $K$ determined in (7). Collecting all terms with the same power of $\varphi\left(\xi_{n}\right)$, the left-hand side of (7) is converted into polynomial in $\varphi\left(\xi_{n}\right)$. Setting each coefficient of this polynomial to be zero, we will derive a set of algebraic equations for $A, a_{i}=$ $(0,1, \ldots, K)$.

Step 5. Solving the overdetermined system of nonlinear algebraic equations by using Mathematica or Maple, we end up with explicit expressions of $A, a_{i}$. 
Step 6. Using the results obtained in above steps, we can finally obtain exact solutions of (5).

\section{Applications of the Modified Truncated Expansion Method}

In this section, we apply the proposed modified truncated expansion method to construct the exact solutions for the nonlinear DDEs via the lattice equation, the discrete nonlinear Schrodinger equation with a saturable nonlinearity, the quintic discrete nonlinear Schrodinger equation, and the relativistic Toda lattice system which are very important in the mathematical physics and have been paid attention by many researchers.

3.1. Example 1: The General Lattice Equation. In this subsection, we use the modified truncated expansion method to find the exact solutions of the general lattice equation. The traveling wave variable (6) permits us converting (1) into the following form:

$$
\begin{array}{r}
C_{1} U^{\prime}\left(\xi_{n}\right)-\left[\alpha+\beta U\left(\xi_{n}\right)+\gamma U^{2}\left(\xi_{n}\right)\right] \\
\times\left[U\left(\xi_{n}+d\right)-U\left(\xi_{n}-d\right)\right]=0,
\end{array}
$$

where $\left(^{\prime}\right)=d / d \xi_{n}$. Considering the homogeneous balance between the highest order derivatives and nonlinear term in (12), we get $K=1$. So we look for the solution of (12) in the following form:

$$
U\left(\xi_{n}\right)=a_{0}+a_{1} \varphi\left(\xi_{n}\right),
$$

where $a_{0}$ and $a_{1}$ are arbitrary constants to be determined later and $\varphi\left(\xi_{n}\right)$ satisfies (9) and (10). We substitute (13), (9), and (10) into (12) and collect all terms with the same power in $\left[\varphi\left(\xi_{n}\right)\right]^{i},(i=0,1,2, \ldots)$. Setting each coefficient of this polynomial to zero, we derive a set of algebraic equations for $a_{0}, a_{1}, d$ and $C_{1}$. Solving the set of algebraic equations by using Maple or Mathematica, we have the following results:

$$
\begin{gathered}
a_{0}=-\frac{1}{2} \frac{\beta e^{d}+\beta \pm \sqrt{-\left(e^{d}-1\right)^{2}\left(-\beta^{2}+4 \alpha \gamma\right)}}{\left(e^{d}+1\right) \gamma}, \\
a_{1}= \pm \frac{A \sqrt{-\left(e^{d}-1\right)^{2}\left(-\beta^{2}+4 \alpha \gamma\right)}}{\left(e^{d}+1\right) \gamma}, \\
C_{1}=\frac{\left(e^{d}-1\right)\left(4 \alpha \gamma-\beta^{2}\right)}{\left(e^{d}+1\right) \gamma},
\end{gathered}
$$

where $\alpha, A, d, \beta$, and $\gamma$ are arbitrary constants. In this case the exact wave solution takes the following form:

$$
\begin{aligned}
U\left(\xi_{n}\right)= & -\frac{1}{2} \frac{\beta e^{d}+\beta \pm \sqrt{-\left(e^{d}-1\right)^{2}\left(-\beta^{2}+4 \alpha \gamma\right)}}{\left(e^{d}+1\right) \gamma} \\
& \pm \frac{A \sqrt{-\left(e^{d}-1\right)^{2}\left(-\beta^{2}+4 \alpha \gamma\right)}}{\left(e^{d}+1\right) \gamma\left(A+e^{\xi_{n}}\right)}
\end{aligned}
$$

where $\xi_{n}=\left(\left(e^{d}-1\right)\left(4 \alpha \gamma-\beta^{2}\right) /\left(e^{d}+1\right) \gamma\right) t+n d+\xi_{0}$.
3.2. Example 2: The Discrete Nonlinear Schrodinger Equation with a Saturable Nonlinearity. If we take the transformation

$$
\psi_{n}=Y\left(\xi_{n}\right) e^{-i(\sigma t+\rho)}, \quad \xi_{n}=d n+\beta,
$$

where $d, \sigma, \beta$, and $\rho$ are arbitrary constants to be determined later.

The transformation (16) leads to write (2) into the following form:

$$
(\sigma-2) Y\left(\xi_{n}\right)+Y\left(\xi_{n}+d\right)+Y\left(\xi_{n}-d\right)+\frac{\eta Y^{3}\left(\xi_{n}\right)}{1+\mu Y^{2}\left(\xi_{n}\right)}=0 .
$$

We suppose the solution of (17) takes the form

$$
Y\left(\xi_{n}\right)=a_{0}+a_{1} \varphi\left(\xi_{n}\right)
$$

where $a_{0}$ and $a_{1}$ are arbitrary constants to be determined later and $\varphi\left(\xi_{n}\right)$ satisfies $(9)$ and $(10)$.

We substitute (18), (9), and (10) into (17) and collect all terms with the same power in $\left[\varphi\left(\xi_{n}\right)\right]^{i},(i=0,1,2, \ldots)$. Setting each coefficient of this polynomial to zero, we derive a set of algebraic equations for $a_{0}, a_{1}, d$, and $C_{1}$. Solving the set of algebraic equations by using Maple or Mathematica, we have the following results:

$$
\begin{gathered}
a_{0}= \pm \frac{\left(e^{\alpha}-1\right)}{\sqrt{-\mu}\left(e^{\alpha}+1\right)}, \quad a_{1}= \pm \frac{2 A\left(1-e^{\alpha}\right)}{\sqrt{-\mu}\left(e^{\alpha}+1\right)}, \\
\sigma=\frac{2\left(e^{\alpha}-1\right)^{2}}{\left(e^{\alpha}+1\right)^{2}}, \quad \eta=\frac{8 e^{\alpha} \mu}{\left(e^{\alpha}+1\right)^{2}},
\end{gathered}
$$

where $\alpha, \mu, A$, and $d$ are arbitrary constants. In this case the exact wave solution of (2) takes the following form:

$$
\begin{aligned}
\psi_{n}= & \left( \pm \frac{\left(e^{\alpha}-1\right)}{\sqrt{-\mu}\left(e^{\alpha}+1\right)} \pm \frac{2 A\left(1-e^{\alpha}\right)}{\sqrt{-\mu}\left(e^{\alpha}+1\right) A+e^{\xi_{n}}}\right) \\
& \times e^{-i\left(\left(2\left(e^{\alpha}-1\right)^{2} /\left(e^{\alpha}+1\right)^{2}\right) t+\rho\right)},
\end{aligned}
$$

where $\xi_{n}=d n+\beta$.

\subsection{Example 3: The Quintic Discrete Nonlinear Schrodinger} Equation. In this subsection, we study the quintic discrete nonlinear Schrodinger equation (3) by using the modified truncated expansion method.

If we take the transformation

$$
\psi_{n}=Y_{n} e^{i \omega t}
$$

where $\omega$ is arbitrary constant to be determined later.

The transformation (21) leads to write (3) into the following form:

$$
Y_{n+1}+Y_{n-1}=\frac{(2 \alpha-\omega) Y_{n}-\beta Y_{n}^{3}}{\alpha+\gamma Y_{n}^{2}+\delta Y_{n}^{4}}
$$

If we suppose

$$
Y_{n}=U\left(\xi_{n}\right), \quad \xi_{n}=d n+k
$$


Equation (23) leads to write (22) into the following form:

$$
U\left(\xi_{n}+d\right)+U\left(\xi_{n}-d\right)=\frac{(2 \alpha-\omega) U\left(\xi_{n}\right)-\beta U^{3}\left(\xi_{n}\right)}{\alpha+\gamma U^{2}\left(\xi_{n}\right)+\delta U^{4}\left(\xi_{n}\right)}
$$

We suppose that the solution of (24) takes the form

$$
U\left(\xi_{n}\right)=a_{0}+a_{1} \varphi\left(\xi_{n}\right)
$$

where $a_{0}$ and $a_{1}$ are arbitrary constants to be determined later and $\varphi\left(\xi_{n}\right)$ satisfies (9) and (10). We substitute (25), (9), and (10) into (24) and collect all terms with the same power in $\left[\varphi\left(\xi_{n}\right)\right]^{i},(i=0,1,2, \ldots)$. Setting each coefficient of this polynomial to zero, we derive a set of algebraic equations for $a_{0}, a_{1}, d$, and $C_{1}$. Solving the set of algebraic equations by using Maple or Mathematica, we have the following results:

$$
\begin{gathered}
a_{0}= \pm \frac{1}{4} \frac{\sqrt{2} \sqrt{\delta e^{d} \beta}\left(e^{d}-1\right)}{\delta e^{d}}, \\
a_{1}= \pm \frac{1}{2} \frac{\sqrt{2} \sqrt{\delta e^{d} \beta}\left(e^{d}-1\right) A}{\delta e^{d}}, \\
\omega=-\frac{\beta}{32} \frac{\left[8 \gamma e^{d}\left(e^{d}-1\right)^{2}+\beta\left(\left(e^{d}\right)^{2}-1\right)^{2}\right]}{\delta\left(e^{d}\right)^{2}}, \\
\alpha=-\frac{\beta}{64} \frac{\left(e^{d}+1\right)^{2}\left(8 \gamma e^{d}+\beta\left(e^{d}+1\right)^{2}\right)}{\delta\left(e^{d}\right)^{2}},
\end{gathered}
$$

where $\beta, A, d, \gamma$, and $\delta$ are arbitrary constants. In this case the solitary wave solution takes the following form:

$$
U\left(\xi_{n}\right)= \pm \frac{1}{4} \frac{\sqrt{2} \sqrt{\delta e^{d} \beta}\left(e^{d}-1\right)}{\delta e^{d}} \pm \frac{1}{2} \frac{\sqrt{2} \sqrt{\delta e^{d} \beta}\left(e^{d}-1\right) A}{\delta e^{d}\left(A+e^{\xi_{n}}\right)}
$$

Consequently the exact solution of (3) is given by

$$
\begin{aligned}
\psi_{n}= & \left( \pm \frac{1}{4} \frac{\sqrt{2} \sqrt{\delta e^{d} \beta}\left(e^{d}-1\right)}{\delta e^{d}} \pm \frac{1}{2} \frac{\sqrt{2} \sqrt{\delta e^{d} \beta}\left(e^{d}-1\right) A}{\delta e^{d}\left(A+e^{\xi_{n}}\right)}\right) \\
& \times e^{i\left(-(\beta / 32)\left(\left[8 \gamma e^{d}\left(e^{d}-1\right)^{2}+\beta\left(\left(e^{d}\right)^{2}-1\right)^{2}\right] / \delta\left(e^{d}\right)^{2}\right)\right) t},
\end{aligned}
$$

where $\xi_{n}=n d+k$.

3.4. Example 4: The Relativistic Toda Lattice System. In this subsection, we use the modified truncated expansion method to study the relativistic Toda lattice system (4). The traveling wave variables $u_{n}=U\left(\xi_{n}\right), v_{n}=V\left(\xi_{n}\right)$, and $\xi_{n}=C_{1} t+n d+\xi_{0}$ permit us to reduce (4) to the following nonlinear difference differential equations:

$$
\begin{aligned}
& C_{1} U^{\prime}\left(\xi_{n}\right)-\left(1+\alpha U\left(\xi_{n}\right)\right) \\
& \times\left(V\left(\xi_{n}\right)-V\left(\xi_{n}-d\right)\right)=0, \\
& C_{1} V^{\prime}\left(\xi_{n}\right)-V\left(\xi_{n}\right)\left(U\left(\xi_{n}+d\right)-U\left(\xi_{n}\right)\right. \\
& \left.\quad+\alpha V\left(\xi_{n}+d\right)-\alpha V\left(\xi_{n}-d\right)\right)=0 .
\end{aligned}
$$

Considering the homogeneous balance between the highest order derivatives and nonlinear terms in (30), we get $k=1$. So we look for the solutions of (30) in the form

$$
U\left(\xi_{n}\right)=a_{0}+a_{1} \varphi\left(\xi_{n}\right), \quad V\left(\xi_{n}\right)=b_{0}+b_{1} \varphi\left(\xi_{n}\right),
$$

where $a_{0}, b_{0}, a_{1}$, and $b_{1}$ are arbitrary constants to be determined later and $\varphi\left(\xi_{n}\right)$ satisfies (9), and (10). We substitute (31), (9) and (10) into (30) and collect all terms with the same power in $\left[\varphi\left(\xi_{n}\right)\right]^{i},(i=0,1,2, \ldots)$. Setting each coefficient of this polynomial to zero, we derive a set of algebraic equations for $a_{0}, a_{1}, d$, and $C_{1}$. Solving the set of algebraic equations by using Maple or Mathematica, we have the following results:

$$
\begin{array}{cc}
a_{0}=-\frac{\left(e^{d}-1\right)+\alpha C_{1}}{\alpha\left(e^{d}-1\right)}, & a_{1}=-C_{1} A, \\
b_{0}=\frac{C_{1}}{\alpha\left(e^{d}-1\right)}, & b_{1}=\frac{C_{1} A}{\alpha},
\end{array}
$$

where $A, d, C_{1}$, and $\alpha$ are arbitrary constants. In this case the solitary wave solutions take the following form:

$$
\begin{aligned}
& U\left(\xi_{n}\right)=-\frac{e^{d}-1+\alpha C_{1}}{\alpha\left(e^{d}-1\right)}+\frac{-C_{1} A}{A+e^{\xi_{n}}}, \\
& V\left(\xi_{n}\right)=\frac{C_{1}}{\alpha\left(e^{d}-1\right)}+\frac{C_{1} A}{\alpha\left(A+e^{\xi_{n}}\right)},
\end{aligned}
$$

where $\xi_{n}=C_{1} t+n d+\xi_{0}$.

\section{Description of the Rational Solitary Wave Functions Method}

In this section, we would like to outline the algorithm for using the rational solitary wave functions method to solve nonlinear DDEs. Consider a given system of $M$ polynomial NDDEs:

$$
\begin{gathered}
\Delta\left(u_{n+p_{1}}(X), \ldots, u_{n+p_{k}}(X), u_{n+p_{1}}^{\prime}(X), \ldots, u_{n+p_{k}}^{\prime}(X), \ldots,\right. \\
\left.u_{n+p_{1}}^{(r)}(X), \ldots, u_{n+p_{k}}^{(r)}(X)\right)=0
\end{gathered}
$$

where the dependent variable $u$ has $M$ components $u_{i}$, the continuous variable $x$ has $N$ components $x_{j}$, the discrete variable $n$ has $Q$ components $n_{i}$, the $k$ shift vectors $P_{s} \in Z^{\mathrm{Q}}$, 
and $u^{(r)}$ denotes the collection of mixed derivative terms of order $r$.

The main steps of the algorithm for the rational solitary wave functions method to solve nonlinear DDEs are outlined as follows.

Step 1. We suppose the wave transformation in the following form:

$$
\begin{array}{r}
u_{n+p_{s}}(X)=U\left(\xi_{n+p_{s}}\right), \quad \xi_{n}=\sum_{i=1}^{Q} d_{i} n_{i}+\sum_{j=1}^{m} c_{j} x_{j}+\xi_{0} \\
s=1,2, \ldots, k
\end{array}
$$

where the coefficients $d_{i}(i=1, \ldots, Q), c_{j}(j=1, \ldots, N)$ and the phase $\xi_{0}$ are constants. The transformations (34) lead to write (33) into the following form:

$$
\begin{aligned}
& \Delta\left(U_{n+p_{1}}\left(\xi_{n}\right), \ldots, U_{n+p_{k}}\left(\xi_{n}\right), U_{n+p_{1}}^{\prime}\left(\xi_{n}\right), \ldots,\right. \\
& \left.\quad U_{n+p_{k}}^{\prime}\left(\xi_{n}\right), \ldots, U_{n+p_{1}}^{(r)}\left(\xi_{n}\right), \ldots, U_{n+p_{k}}^{(r)}\left(\xi_{n}\right)\right)=0 .
\end{aligned}
$$

Step 2. We suppose the rational solitary wave series expansion solutions of (35) in the following form:

$$
U\left(\xi_{n}\right)=\sum_{i=0}^{N} a_{i}\left[g\left(\xi_{n}\right)\right]^{i}+\sum_{j=1}^{N} b_{j}\left[g\left(\xi_{n}\right)\right]^{j-1} f\left(\xi_{n}\right),
$$

with

$$
\begin{aligned}
& f\left(\xi_{n}\right)=\frac{1}{A \tanh \left(\xi_{n}\right)+B \operatorname{sech}\left(\xi_{n}\right)}, \\
& g\left(\xi_{n}\right)=\frac{\operatorname{sech}\left(\xi_{n}\right)}{A \tanh \left(\xi_{n}\right)+B \operatorname{sech}\left(\xi_{n}\right)},
\end{aligned}
$$

which satisfy

$$
\begin{gathered}
f^{\prime}\left(\xi_{n}\right)=-A g^{2}\left(\xi_{n}\right)+\frac{B g\left(\xi_{n}\right)}{A}\left[1-B g\left(\xi_{n}\right)\right] \\
g^{\prime}\left(\xi_{n}\right)=-A f\left(\xi_{n}\right) g\left(\xi_{n}\right) \\
f^{2}\left(\xi_{n}\right)=g^{2}\left(\xi_{n}\right)+\frac{1}{A^{2}}\left[1-B g\left(\xi_{n}\right)\right]^{2} \\
f\left(\xi_{n} \pm \sigma_{s}\right) \\
=\left(A^{2} f\left(\sigma_{s}\right) f\left(\xi_{n}\right) \pm\left[1-B g\left(\xi_{n}\right)\right]\left[1-B g\left(\sigma_{s}\right)\right]\right) \\
\quad \times\left(A^{2} f\left(\sigma_{s}\right)\left[1-B g\left(\xi_{n}\right)\right] \pm A^{2} f\left(\xi_{n}\right)\left[1-B g\left(\sigma_{s}\right)\right]\right. \\
\left.\quad+B A^{2} g\left(\xi_{n}\right) g\left(\sigma_{s}\right)\right)^{-1}, \\
\left.g\left(\xi_{n} \pm \sigma_{s}\right) \quad \times\left[1-B g\left(\sigma_{s}\right)\right]+B g\left(\xi_{n}\right) g\left(\sigma_{s}\right)\right)^{-1} \\
=\left(g\left(\xi_{n}\right) g\left(\sigma_{s}\right)\right)\left(f\left(\sigma_{s}\right)\left[1-B g\left(\xi_{n}\right)\right] \pm f\left(\xi_{n}\right)\right.
\end{gathered}
$$

where $a_{i}, b_{j}, A$, and $B$ are arbitrary constants to be determined later and

$$
\sigma_{s}=p_{s_{1}} d_{1}+p_{s_{2}} d_{2}+\cdots+p_{s Q} d_{Q}
$$

Also, we can assume that

$$
\begin{aligned}
& f\left(\xi_{n}\right)=\frac{1}{A \tan \left(\xi_{n}\right)+B \sec \left(\xi_{n}\right)}, \\
& g\left(\xi_{n}\right)=\frac{\sec \left(\xi_{n}\right)}{A \tan \left(\xi_{n}\right)+B \sec \left(\xi_{n}\right)},
\end{aligned}
$$

which satisfy

$$
\begin{gathered}
f^{\prime}\left(\xi_{n}\right)=-A g^{2}\left(\xi_{n}\right)-\frac{B g\left(\xi_{n}\right)}{A}\left[1-B g\left(\xi_{n}\right)\right] \\
g^{\prime}\left(\xi_{n}\right)=-A f\left(\xi_{n}\right) g\left(\xi_{n}\right) \\
f^{2}\left(\xi_{n}\right)=g^{2}\left(\xi_{n}\right)-\frac{1}{A^{2}}\left[1-B g\left(\xi_{n}\right)\right]^{2} \\
f\left(\xi_{n} \pm \sigma_{s}\right) \\
=\left(A^{2} f\left(\sigma_{s}\right) f\left(\xi_{n}\right) \mp\left[1-B g\left(\xi_{n}\right)\right]\left[1-B g\left(\sigma_{s}\right)\right]\right) \\
\times\left(A^{2} f\left(\sigma_{s}\right)\left[1-B g\left(\xi_{n}\right)\right] \pm A^{2} f\left(\xi_{n}\right)\left[1-B g\left(\sigma_{s}\right)\right]\right. \\
\left.\quad+B A^{2} g\left(\xi_{n}\right) g\left(\sigma_{s}\right)\right)^{-1}, \\
g\left(\xi_{n} \pm \sigma_{s}\right) \quad\left(g\left(\xi_{n}\right) g\left(\sigma_{s}\right)\right)\left(f\left(\sigma_{s}\right)\left[1-B g\left(\xi_{n}\right)\right] \pm f\left(\xi_{n}\right)\right. \\
\left.\times\left[1-B g\left(\sigma_{s}\right)\right]+B g\left(\xi_{n}\right) g\left(\sigma_{s}\right)\right)^{-1}
\end{gathered}
$$

Equations (38) and (40) can be written into unified form:

$$
\begin{gathered}
f^{\prime}\left(\xi_{n}\right)=-A g^{2}\left(\xi_{n}\right)+\rho \frac{B g\left(\xi_{n}\right)}{A}\left[1-B g\left(\xi_{n}\right)\right] \\
g^{\prime}\left(\xi_{n}\right)=-A f\left(\xi_{n}\right) g\left(\xi_{n}\right) \\
f^{2}\left(\xi_{n}\right)=g^{2}\left(\xi_{n}\right)+\rho \frac{1}{A^{2}}\left[1-B g\left(\xi_{n}\right)\right]^{2} \\
f\left(\xi_{n} \pm \sigma_{s}\right) \\
=\left(A^{2} f\left(\sigma_{s}\right) f\left(\xi_{n}\right) \pm \rho\left[1-B g\left(\xi_{n}\right)\right]\left[1-B g\left(\sigma_{s}\right)\right]\right) \\
\times\left(A^{2} f\left(\sigma_{s}\right)\left[1-B g\left(\xi_{n}\right)\right] \pm A^{2} f\left(\xi_{n}\right)\left[1-B g\left(\sigma_{s}\right)\right]\right. \\
\left.\quad+B A^{2} g\left(\xi_{n}\right) g\left(\sigma_{s}\right)\right)^{-1}, \\
\left.g\left(\xi_{n} \pm \sigma_{s}\right) \quad \times\left[1-B g\left(\sigma_{s}\right)\right]+B g\left(\xi_{n}\right) g\left(\sigma_{s}\right)\right)^{-1} \\
=\left(g\left(\xi_{n}\right) g\left(\sigma_{s}\right)\right)\left(f\left(\sigma_{s}\right)\left[1-B g\left(\xi_{n}\right)\right] \pm f\left(\xi_{n}\right)\right.
\end{gathered}
$$

where $\rho= \pm 1$. 
Step 3. Determine the degree $N$ of (35) by balancing the highest order nonlinear term(s) and the highest order derivatives of $U\left(\xi_{n}\right)$ in (35).

Step 4. Substituting (36)-(41) and given the value of $N$ determined in Step 3 into (35) and collecting all terms with the same degree of $f\left(\xi_{n}\right)$ and $g\left(\xi_{n}\right)$ together, the left-hand side of (35) is converted into polynomial in $f\left(\xi_{n}\right)$ and $g\left(\xi_{n}\right)$. Then setting each coefficient $f^{i}\left(\xi_{n}\right), g^{j}\left(\xi_{n}\right)(i=0,1, j=$ $0,1,2, \ldots)$ of this polynomial to zero, we derive a set of algebraic equations for $a_{i}, b_{j}, C_{i}, A, B$.

Step 5. Solving the overdetermined system of nonlinear algebraic equations by using Maple or Mathematica software package, we end up with explicit expressions for $a_{i}, b_{j}, C_{i}, A$, and $B$.

Step 6. Substituting $a_{i}, b_{j}, C_{i}, A$, and $B$ into (36) along with (37) and (39), we can finally obtain the rational solitary wave solutions for nonlinear difference differential equations (33).

\section{Applications}

In this section, we apply the proposed rational solitary wave functions method to construct the rational solitary wave solutions for some nonlinear DDEs via the discrete nonlinear Schrodinger equation with a saturable nonlinearity, the quintic discrete nonlinear Schrodinger equation, and the relativistic Toda lattice system, which are very important in the mathematical physics and modern physics.

5.1. Example 1: The Discrete Nonlinear Schrodinger Equation with a Saturable Nonlinearity. We suppose that the solution of (17) takes the form

$$
U\left(\xi_{n}\right)=a_{0}+a_{1} f\left(\xi_{n}\right)+a_{2} g\left(\xi_{n}\right),
$$

where $a_{0}, a_{1}$, and $b_{1}$ are arbitrary constants to be determined later. With the aid of Maple, we substitute (43), (41) into (17) and collect all terms with the same power in $f^{i}\left(\xi_{n}\right), g^{j}\left(\xi_{n}\right)(i=0,1, j=0,1,2, \ldots)$. Setting each coefficient of these terms $f^{i}\left(\xi_{n}\right), g^{j}\left(\xi_{n}\right)(i=0,1, j=$ $0,1,2, \ldots)$ to zero yields a set of algebraic equations which have the following solutions.

Case $1(\rho=1)$.

$$
\begin{gathered}
a_{1}= \pm A \sqrt{\frac{-2}{\eta} \frac{\sinh (d / 2)}{\cosh ^{2}(d / 2)}} \\
a_{2}= \pm \sqrt{\frac{-2\left(A^{2}+B^{2}\right)}{\eta}} \frac{\sinh (d / 2)}{\cosh ^{2}(d / 2)} \\
\mu=\frac{1}{4}(1+\cosh (d)) \eta \\
\sigma=\frac{2(\cosh (d)-1)}{1+\cos (d)}, \quad a_{0}=0
\end{gathered}
$$

where $A, B$, and $\eta$ are arbitrary constants and $\eta<0$. In this case the rational hyperbolic solitary wave solution of (17) takes the following form:

$$
\begin{aligned}
U\left(\xi_{n}\right)= & \pm A \sqrt{\frac{-2}{\eta} \frac{\sinh (d / 2)}{\cosh ^{2}(d / 2)\left[A \tanh \left(\xi_{n}\right)+B \operatorname{sech}\left(\xi_{n}\right)\right]}} \\
& \pm \sqrt{\frac{-2\left(A^{2}+B^{2}\right)}{\eta}} \frac{\sinh (d / 2) \operatorname{sech}\left(\xi_{n}\right)}{\left[A \tanh \left(\xi_{n}\right)+B \operatorname{sech}\left(\xi_{n}\right)\right]} .
\end{aligned}
$$

Consequently the rational hyperbolic solitary wave solution of (2) has the following form:

$$
\begin{aligned}
\psi_{n}= & {\left[= \pm A \sqrt{\frac{-2}{\eta} \frac{\sinh (d / 2)}{\cosh ^{2}(d / 2)\left[A \tanh \left(\xi_{n}\right)+B \operatorname{sech}\left(\xi_{n}\right)\right]}}\right.} \\
& \pm \sqrt{\left.\frac{-2\left(A^{2}+B^{2}\right)}{\eta} \frac{\sinh (d / 2) \operatorname{sech}\left(\xi_{n}\right)}{\left[A \tanh \left(\xi_{n}\right)+B \operatorname{sech}\left(\xi_{n}\right)\right]}\right]} \\
& \times e^{-i((2(\cosh (d)-1) /(1+\cos (d))) t+\rho)},
\end{aligned}
$$

where $\xi_{n}=n d+\beta$.

Case $2(\rho=-1)$.

$$
\begin{gathered}
a_{1}= \pm A \sqrt{\frac{-2}{\eta} \frac{\sin (d / 2)}{\cos ^{2}(d / 2)},} \\
a_{2}= \pm \sqrt{\frac{-2\left(A^{2}-B^{2}\right)}{\eta} \frac{\sin (d / 2)}{\cos ^{2}(d / 2)}} \\
\mu=\frac{1}{4}(\cos (d)+1) \eta, \\
\sigma=\frac{2(-1+2 \cos (d))}{\cos (d)+1}, \quad a_{0}=0,
\end{gathered}
$$

where $A, B$, and $\eta$ are arbitrary constants and $\eta<0$. In this case the rational trigonometric solitary wave solution of (17) takes the following form:

$$
\begin{aligned}
U\left(\xi_{n}\right)= & \pm A \sqrt{\frac{-2}{\eta} \frac{\sin (d / 2)}{\cos ^{2}(d / 2)\left[A \tan \left(\xi_{n}\right)+B \sec \left(\xi_{n}\right)\right]}} \\
& \pm \sqrt{\frac{-2\left(A^{2}-B^{2}\right)}{\eta}} \\
& \times \frac{\sin (d / 2) \sec \left(\xi_{n}\right)}{\cos ^{2}(d / 2)\left[A \tan \left(\xi_{n}\right)+B \sec \left(\xi_{n}\right)\right]} .
\end{aligned}
$$


Consequently the rational trigonometric solitary wave solution of (2) has the following form:

$$
\begin{aligned}
\psi_{n}=\left[ \pm A \sqrt{\frac{-2}{\eta} \frac{\sin (d / 2)}{\cos ^{2}(d / 2)\left[A \tan \left(\xi_{n}\right)+B \sec \left(\xi_{n}\right)\right]}}\right. \\
\quad \pm \sqrt{\frac{-2\left(A^{2}-B^{2}\right)}{\eta}} \\
\left.\quad \times \frac{\sin (d / 2) \sec \left(\xi_{n}\right)}{\cos ^{2}(d / 2)\left[A \tan \left(\xi_{n}\right)+B \sec \left(\xi_{n}\right)\right]}\right] \\
\times e^{-i((2(-1+2 \cos (d)) /(\cos (d)+1)) t+\rho)},
\end{aligned}
$$

where $\xi_{n}=n d+\beta$.

\subsection{Example 2: The Quintic Discrete Nonlinear Schrodinger} Equation. In this subsection, we study the quintic discrete nonlinear Schrodinger equation (3) by using the rational solitary wave functions method.

We suppose that the solution of (24) takes the form

$$
U\left(\xi_{n}\right)=a_{0}+a_{1} f\left(\xi_{n}\right)+a_{2} g\left(\xi_{n}\right),
$$

where $a_{0}, a_{1}$, and $b_{1}$ are arbitrary constants to be determined later. With the aid of Maple, we substitute (50) and (41) into (24), collect all terms with the same power in $f^{i}\left(\xi_{n}\right), g^{j}\left(\xi_{n}\right)(i=0,1, j=0,1,2, \ldots)$, and setting each coefficient of these terms $f^{i}\left(\xi_{n}\right), g^{j}\left(\xi_{n}\right)(i=0,1, j=$ $0,1,2, \ldots)$ to zero yields a set of algebraic equations which have the following solutions.

Case $1(\rho=1)$.

$$
\begin{aligned}
& a_{1}= \pm \frac{1}{4}((\sqrt{2} \sqrt{\delta \beta(\cosh (d)+\sinh (d))} \\
& \times(\cosh (d)+\sin h(d)-1) A) \\
& \left.\times(\delta(\cosh (d)+\sin h(d)))^{-1}\right), \\
& a_{2}= \pm \frac{1}{4}\left(\left(\sqrt{2} \sqrt{\delta \beta\left(A^{2}+B^{2}\right)(\cosh (d)+\sin h(d))}\right.\right. \\
& \times(\cosh (d)+\sin h(d)-1)) \\
& \left.\times(\delta(\cosh (d)+\sin h(d)))^{-1}\right), \\
& \alpha=\frac{-1}{16} \frac{\left[4 \gamma(\cosh (d)+1)+\beta\left(1+2 \cosh (d)+\cosh ^{2}(d)\right)\right]}{\cosh (d)-1}, \\
& \omega=\frac{-1}{8} \frac{\beta\left[4 \gamma(\cosh (d)-1)+\beta\left(\cosh ^{2}(d)-1\right)\right]}{\delta}, \\
& a_{0}=0 \text {, }
\end{aligned}
$$

where $A, B, \beta, \gamma$, and $\delta$ are arbitrary constants. In this case the rational hyperbolic solitary wave solution of (24) takes the following form:

$$
\begin{aligned}
U\left(\xi_{n}\right)= \pm \frac{1}{4}(( & \sqrt{2} \sqrt{\delta \beta(\cos h(d)+\sin h(d))} \\
& \times(\cosh (d)+\sin h(d)-1) A) \\
\times & (\delta(\cos h(d)+\sinh (d)) \\
& \left.\left.\times\left(A \tanh \left(\xi_{n}\right)+B \operatorname{sech}\left(\xi_{n}\right)\right)\right)^{-1}\right) \\
\pm \frac{1}{4}( & \sqrt{2} \sqrt{\delta \beta\left(A^{2}+B^{2}\right)(\cos h(d)+\sin h(d))} \\
& \left.\times(\cosh (d)+\sin h(d)-1) \operatorname{sech}\left(\xi_{n}\right)\right) \\
\times & (\delta(\cos h(d)+\sin h(d)) \\
& \left.\left.\times\left[A \tanh \left(\xi_{n}\right)+B \operatorname{sech}\left(\xi_{n}\right)\right]\right)^{-1}\right) .
\end{aligned}
$$

Consequently the rational hyperbolic solitary wave solution of (3) has the following form:

$$
\begin{aligned}
& \psi_{n}=\left[ \pm \frac{1}{4}((\right. \sqrt{2} \sqrt{\delta \beta(\cos h(d)+\sin h(d))} \\
& \times(\cos h(d)+\sin h(d)-1) A) \\
& \times(\delta(\cos h(d)+\sin h(d)) \\
&\left.\left.\times\left(A \tanh \left(\xi_{n}\right)+B \operatorname{sech}\left(\xi_{n}\right)\right)\right)^{-1}\right) \\
& \pm \frac{1}{4}( \sqrt{2} \sqrt{\delta \beta\left(A^{2}+B^{2}\right)(\cos h(d)+\sin h(d))} \\
& \times\left.(\cos h(d)+\sin h(d)-1) \operatorname{sech}\left(\xi_{n}\right)\right) \\
& \times(\delta(\cos h(d)+\sin h(d)) \\
&\left.\left.\left.\times\left[A \tanh \left(\xi_{n}\right)+B \operatorname{sech}\left(\xi_{n}\right)\right]\right)^{-1}\right)\right] \\
& \times e^{i\left((-1 / 8)\left(\beta\left[4 \gamma(\cos h(d)-1)+\beta\left(\cosh { }^{2}(d)-1\right)\right] / \delta\right)\right) t},
\end{aligned}
$$

where $\xi_{n}=n d+k$.

Case $2(\rho=-1)$.

$$
\begin{aligned}
a_{1} & = \pm \frac{1}{2} \frac{\sqrt{-\delta \beta(\cos (d)-1)} A}{\delta}, \\
a_{2} & = \pm \frac{1}{2} \frac{\sqrt{\delta \beta\left(A^{2}-B^{2}\right)(1-\cos (d))}}{\delta},
\end{aligned}
$$




$$
\begin{gathered}
a_{0}=0, \\
\alpha=\frac{-1}{16} \frac{\beta\left[(\cos (d)+1)(4 \gamma+2 \beta)-\beta \sin ^{2}(d)\right]}{\delta}, \\
\omega=\frac{-1}{8} \frac{\beta\left[4 \gamma(\cos (d)-1)-\beta \sin ^{2}(d)\right]}{\delta},
\end{gathered}
$$

where $A, B, \beta, \gamma$, and $\delta$ are arbitrary constants. In this case the rational trigonometric solitary wave solution of (24) takes the following form:

$$
\begin{aligned}
U\left(\xi_{n}\right)= & \pm \frac{1}{2} \frac{\sqrt{-\delta \beta(\cos (d)-1)} A}{\delta\left(A \tan \left(\xi_{n}\right)+B \sec \left(\xi_{n}\right)\right)} \\
& \pm \frac{1}{2} \frac{\sqrt{\delta \beta\left(A^{2}-B^{2}\right)(1-\cos (d))} \sec \left(\xi_{n}\right)}{\delta\left[A \tan \left(\xi_{n}\right)+B \sec \left(\xi_{n}\right)\right]} .
\end{aligned}
$$

Consequently the rational trigonometric solitary wave solution of (3) has the following form:

$$
\begin{gathered}
\psi_{n}=\left( \pm \frac{1}{2} \frac{\sqrt{-\delta \beta(\cos (d)-1)} A}{\delta\left(A \tan \left(\xi_{n}\right)+B \sec \left(\xi_{n}\right)\right)}\right. \\
\left. \pm \frac{1}{2} \frac{\sqrt{\delta \beta\left(A^{2}-B^{2}\right)(1-\cos (d))} \sec \left(\xi_{n}\right)}{\delta\left[A \tan \left(\xi_{n}\right)+B \sec \left(\xi_{n}\right)\right]}\right) \\
\times e^{i\left((-1 / 8)\left(\beta\left[4 \gamma(\cos (d)-1)-\beta \sin ^{2}(d)\right] / \delta\right)\right) t},
\end{gathered}
$$

where $\xi_{n}=n d+k$.

5.3. Example 3: The Relativistic Toda Lattice System. In this subsection, we study the relativistic Toda lattice system (4) by using the rational solitary wave functions method.

If we take the transformation

$$
v_{n}=-\frac{1}{\alpha} u_{n}-\frac{1}{\alpha^{2}}
$$

the transformation (57) reduced the relativistic Toda lattice system (4) into the following difference differential equation:

$$
u_{n_{t}}=\left(u_{n}+\frac{1}{\alpha}\right)\left(u_{n-1}-u_{n}\right)
$$

According to the main steps of rational solitary wave functions method, we seek traveling wave solutions of (58) in the following form:

$$
u_{n}(t)=U\left(\xi_{n}\right), \quad \xi_{n}=C_{1} t+n d+\xi_{0},
$$

where $d, C_{1}$, and $\xi_{0}$ are constants. The transformation (59) permits us converting (58) into the following form:

$$
C_{1} U^{\prime}\left(\xi_{n}\right)=\left(U\left(\xi_{n}\right)+\frac{1}{\alpha}\right)\left[U\left(\xi_{n}-d\right)-U\left(\xi_{n}\right)\right]
$$

Considering the homogeneous balance between the highest order derivatives and nonlinear terms in (60), we get $N=1$. Thus, the solution of (60) has the following form:

$$
U\left(\xi_{n}\right)=a_{0}+a_{1} f\left(\xi_{n}\right)+a_{2} g\left(\xi_{n}\right)
$$

where $a_{0}, b_{0}, a_{1}$, and $b_{1}$ are arbitrary constants to be determined later. With the aid of Maple, we substitute (61) and (41) into (60) and collect all terms with the same power in $f^{i}\left(\xi_{n}\right), g^{j}\left(\xi_{n}\right)(i=0,1, j=0,1,2, \ldots)$. Setting each coefficient of these terms $f^{i}\left(\xi_{n}\right), g^{j}\left(\xi_{n}\right)(i=0,1, j=$ $0,1,2, \ldots)$ to be zero yields a set of algebraic equations which have the following solutions.

Case $1(\rho=1)$.

$$
\begin{gathered}
a_{0}=\frac{1}{\alpha\left(e^{d}-1\right)}\left[\frac{ \pm \alpha a_{2}\left(e^{d}+1\right)}{\sqrt{A^{2}+B^{2}}}+\left(1-e^{d}\right)\right], \\
a_{1}=\frac{ \pm A a_{2}}{\sqrt{A^{2}+B^{2}}}, \quad C_{1}=\frac{ \pm 2 a_{2}}{\sqrt{A^{2}+B^{2}}},
\end{gathered}
$$

where $A, B, a_{2}$, and $\alpha$ are arbitrary constants. In this case the rational hyperbolic solitary wave solution of (58) has the following form:

$$
\begin{aligned}
U\left(\xi_{n}\right)= & \frac{1}{\alpha\left(e^{d}-1\right)}\left[\frac{ \pm \alpha a_{2}\left(e^{d}+1\right)}{\sqrt{A^{2}+B^{2}}}+\left(1-e^{d}\right)\right] \\
& \pm \frac{A a_{2}}{\sqrt{A^{2}+B^{2}}\left[A \tanh \left(\xi_{n}\right)+B \operatorname{sech}\left(\xi_{n}\right)\right]} \\
& +\frac{a_{2} \operatorname{sech}\left(\xi_{n}\right)}{\left[A \tanh \left(\xi_{n}\right)+B \operatorname{sech}\left(\xi_{n}\right)\right]},
\end{aligned}
$$

where

$$
\xi_{n}= \pm \frac{2 a_{2}}{\sqrt{A^{2}+B^{2}}} t+n d+\xi_{0} .
$$

Case $2(\rho=-1)$.

$$
\begin{gathered}
a_{0}=\frac{1}{\alpha \sin (d)}\left[\frac{ \pm \alpha a_{2}(\cos (d)+1)}{\sqrt{A^{2}-B^{2}}}+\sin (d)\right], \\
a_{1}=\frac{ \pm A a_{2}}{\sqrt{A^{2}-B^{2}}}, \quad C_{1}=\frac{ \pm 2 a_{2}}{\sqrt{A^{2}-B^{2}}} .
\end{gathered}
$$

In this case the rational trigonometric solitary wave solution of (58) has the following form:

$$
\begin{aligned}
U\left(\xi_{n}\right)= & \frac{1}{\alpha \sin (d)}\left[\frac{ \pm \alpha a_{2}(\cos (d)+1)}{\sqrt{A^{2}-B^{2}}}+\sin (d)\right] \\
& \pm \frac{A a_{2}}{\sqrt{A^{2}-B^{2}}\left[A \tan \left(\xi_{n}\right)+B \sec \left(\xi_{n}\right)\right]} \\
& +\frac{a_{2} \sec \left(\xi_{n}\right)}{\left[A \tan \left(\xi_{n}\right)+B \sec \left(\xi_{n}\right)\right]}
\end{aligned}
$$

where

$$
\xi_{n}= \pm \frac{2 a_{2}}{\sqrt{A^{2}-B^{2}}} t+n d+\xi_{0} .
$$




\section{Discussion}

When we compare between the results which obtained in this paper and other exact solutions we get the following.

The solutions obtained in the modified truncated expansion functions method are equivalent to the solution obtained by the exp-functions method, but the modified truncated expansion is simple and allowed us to solve more complicated nonlinear difference differential equations such as the discrete nonlinear Schrodinger equation with a saturable nonlinearity, the quintic discrete nonlinear Schrodinger equation, and the relativistic Toda lattice system. For example, the solution (15) equivalent is to the solution (40) in [20].

In the special case when $B=0$ in the rational solitary wave function method, we get this method which is equivalent to the tanh-function method which discussed in [29]. The rational solitary wave function method is extended to the new rational formal solution which is discussed by [30] when $b_{j}=0$ in (36).

Remarks. These methods which are discussed in this paper allowed us to obtain some new rational solitary wave solutions for some complicated nonlinear differential difference equations.

These methods prefer to another methods to convert the complicated rational methods into a direct nonrational method.

\section{Conclusions}

In this paper, we use the modified truncated expansion method to obtain the exact solutions for some nonlinear differential difference equations in the mathematical physics. Also, we calculate the rational solitary wave solutions for the nonlinear differential difference equations. As a result, many new and more rational solitary wave solutions are obtained, from the hyperbolic function solutions and trigonometric function.

\section{Acknowledgment}

The authors wish to thank the referees for their suggestions and very useful comments.

\section{References}

[1] E. Fermi, J. Pasta, and S. Ulam, Collected Papers of Enrico Fermi II, University of Chicago Press, Chicago, Ill, USA, 1965.

[2] W. P. Su, J. R. Schrieffer, and A. J. Heeger, "Solitons in polyacetylene," Physical Review Letters, vol. 42, no. 25, pp. 16981701, 1979.

[3] A. S. Davydov, "The theory of contraction of proteins under their excitation," Journal of Theoretical Biology, vol. 38, no. 3, pp. 559-569, 1973.

[4] P. Marquié, J. M. Bilbault, and M. Remoissenet, "Observation of nonlinear localized modes in an electrical lattice," Physical Review E, vol. 51, no. 6, pp. 6127-6133, 1995.

[5] M. Toda, Theory of Nonlinear Lattices, Springer, Berlin, Germany, 1989.
[6] M. Wadati, "Transformation theories for nonlinear discrete systems," Progress of Theoretical Physics, vol. 59, pp. 36-63, 1976.

[7] Y. Ohta and R. Hirota, "A discrete KdV equation and its Casorati determinant solution," Journal of the Physical Society of Japan, vol. 60, p. 2095, 1991.

[8] M. J. Ablowitz and J. F. Ladik, "Nonlinear differential-difference equations," Journal of Mathematical Physics, vol. 16, no. 3, pp. 598-603, 1974.

[9] X. B. Hu and W. X. Ma, "Application of Hirota's bilinear formalism to the Toeplitz lattice-some special soliton-like solutions," Physics Letters Section A, vol. 293, no. 3-4, pp. 161$165,2002$.

[10] D. Baldwin, U. Göktaş, and W. Hereman, "Symbolic computation of hyperbolic tangent solutions for nonlinear differentialdifference equations," Computer Physics Communications, vol. 162, no. 3, pp. 203-217, 2004.

[11] S. K. Liu, Z. T. Fu, Z. G. Wang, and S. D. Liu, "Periodic solutions for a class of nonlinear differential-difference equations," Communications in Theoretical Physics, vol. 49, no. 5, pp. 1155-1158, 2008.

[12] C. Qiong and L. Bin, "Applications of jacobi elliptic function expansion method for nonlinear differential-difference equations," Communications in Theoretical Physics, vol. 43, no. 3, pp. 385-388, 2005.

[13] F. Xie, M. Ji, and H. Zhao, "Some solutions of discrete sineGordon equation," Chaos, Solitons and Fractals, vol. 33, no. 5, pp. 1791-1795, 2007.

[14] S. D. Zhu, "Exp-function method for the Hybrid-Lattice system," International Journal of Nonlinear Sciences and Numerical Simulation, vol. 8, no. 3, pp. 461-464, 2007.

[15] I. Aslan, "A discrete generalization of the extended simplest equation method," Communications in Nonlinear Science and Numerical Simulation, vol. 15, no. 8, pp. 1967-1973, 2010.

[16] P. Yang, Y. Chen, and Z. B. Li, "ADM-Padé technique for the nonlinear lattice equations," Applied Mathematics and Computation, vol. 210, no. 2, pp. 362-375, 2009.

[17] S. D. Zhu, Y. M. Chu, and S. L. Qiu, "The homotopy perturbation method for discontinued problems arising in nanotechnology," Computers and Mathematics with Applications, vol. 58, no. 11-12, pp. 2398-2401, 2009.

[18] F. Xie, "New travelling wave solutions of the generalized coupled Hirota-Satsuma KdV system," Chaos, Solitons and Fractals, vol. 20, no. 5, pp. 1005-1012, 2004.

[19] E. M. E. Zayed, A. M. Abourabia, K. A. Gepreel, and M. M. El Horbaty, "On the rational solitary wave solutions for the nonlinear Hirota-Satsuma coupled KdV system," Applicable Analysis, vol. 85, pp. 751-768, 2006.

[20] C. S. Liu, "Exponential function rational expansion method for nonlinear differential-difference equations," Chaos, Solitons and Fractals, vol. 40, no. 2, pp. 708-716, 2009.

[21] S. Zhang, L. Dong, J. M. Ba, and Y. N. Sun, “The $\left(\operatorname{frac}\left(G^{\prime} / G\right)\right)$ expansion method for nonlinear differential-difference equations," Physics Letters Section A, vol. 373, no. 10, pp. 905-910, 2009.

[22] I. Aslan, "The Discrete $\left(G^{\prime} / G\right)$-expansion method applied to the differential-difference Burgers equation and the relativistic Toda lattice system," Numerical Methods for Partial Differential Equations, vol. 28, pp. 127-137, 2012.

[23] I. Aslan, "Exact and explicit solutions to the discrete nonlinear Schrödinger equation with asaturable nonlinearity," Physics Letters A, vol. 375, pp. 4214-4217, 2011. 
[24] K. A. Gepreel, "Rational Jacobi Elliptic solutions for nonlinear difference differential equations," Nonlinear Science Letters A, vol. 2, pp. 151-158, 2011.

[25] K. A. Gepreel and A. R. Shehata, "Rational Jacobi elliptic solutions for nonlinear differential-difference lattice equations," Applied Mathematics Letters, vol. 25, pp. 1173-1178, 2012.

[26] K. A. Gepreel, T. A. Nofal, and A. A. AlThobaiti, "The modified rational Jacobi elliptic solutions for nonlinear differential difference equations," Journal of Applied Mathematics, vol. 2012, Article ID 427479, 30 pages, 2012.

[27] G. C. Wu and T. C. Xia, "A new method for constructing soliton solutions to differential-difference equation with symbolic computation," Chaos, Solitons and Fractals, vol. 39, no. 5, pp. 22452248, 2009.

[28] J. Zhang, Z. Liu, S. Li, and M. Wang, "Solitary waves and stable analysis for the quintic discrete nonlinear Schrödinger equation," Physica Scripta, vol. 86, pp. 015401-015409, 2012.

[29] F. Xie and J. Wang, "A new method for solving nonlinear differential-difference equation," Chaos, Solitons and Fractals, vol. 27, no. 4, pp. 1067-1071, 2006.

[30] Q. Wang and Y. Yu, "New rational formal solutions for $(1+1)$ dimensional Toda equation and another Toda equation," Chaos, Solitons and Fractals, vol. 29, no. 4, pp. 904-915, 2006. 


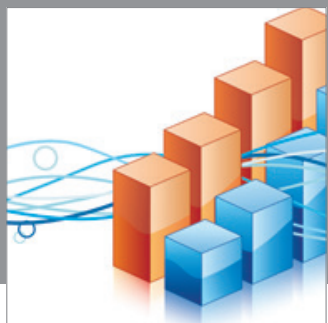

Advances in

Operations Research

mansans

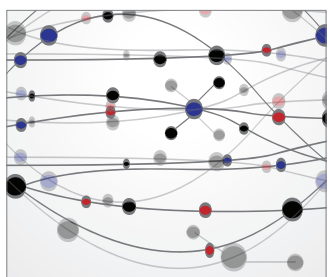

The Scientific World Journal
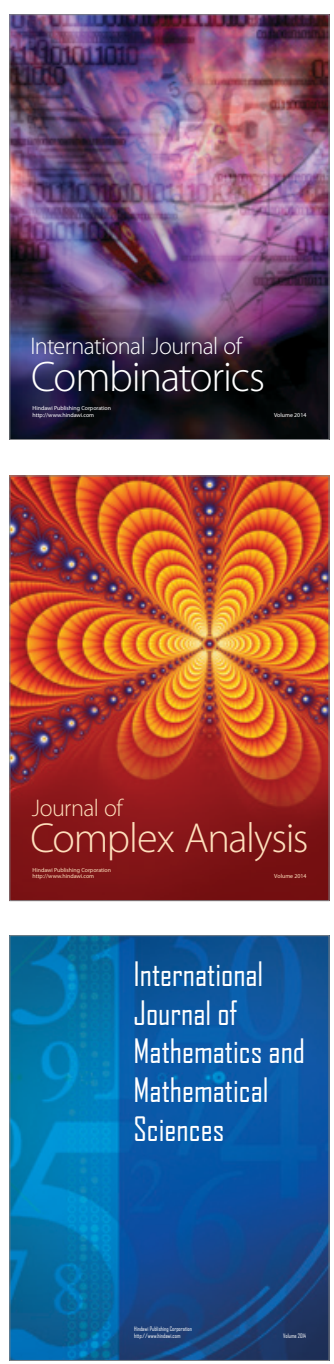
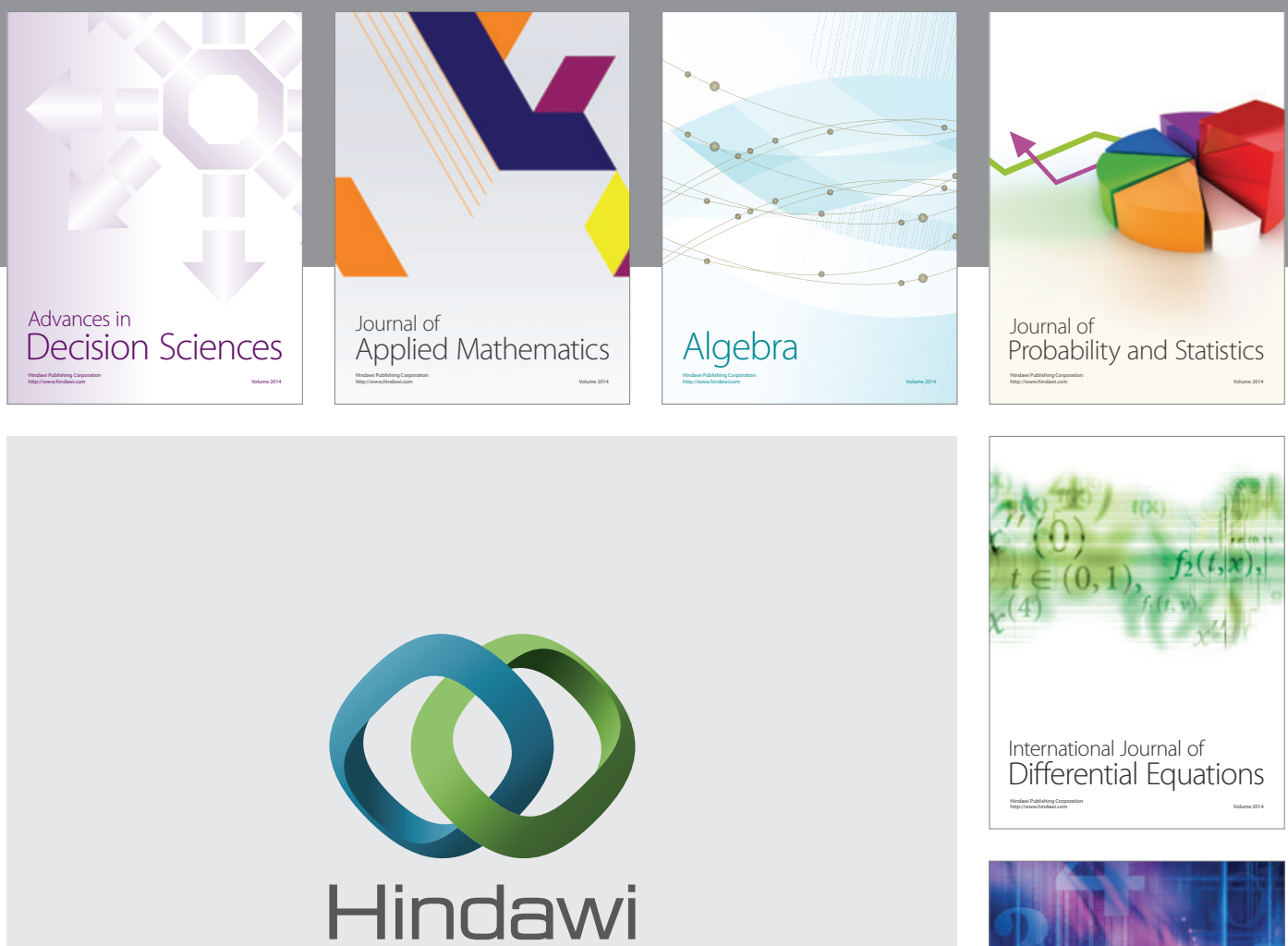

Submit your manuscripts at http://www.hindawi.com
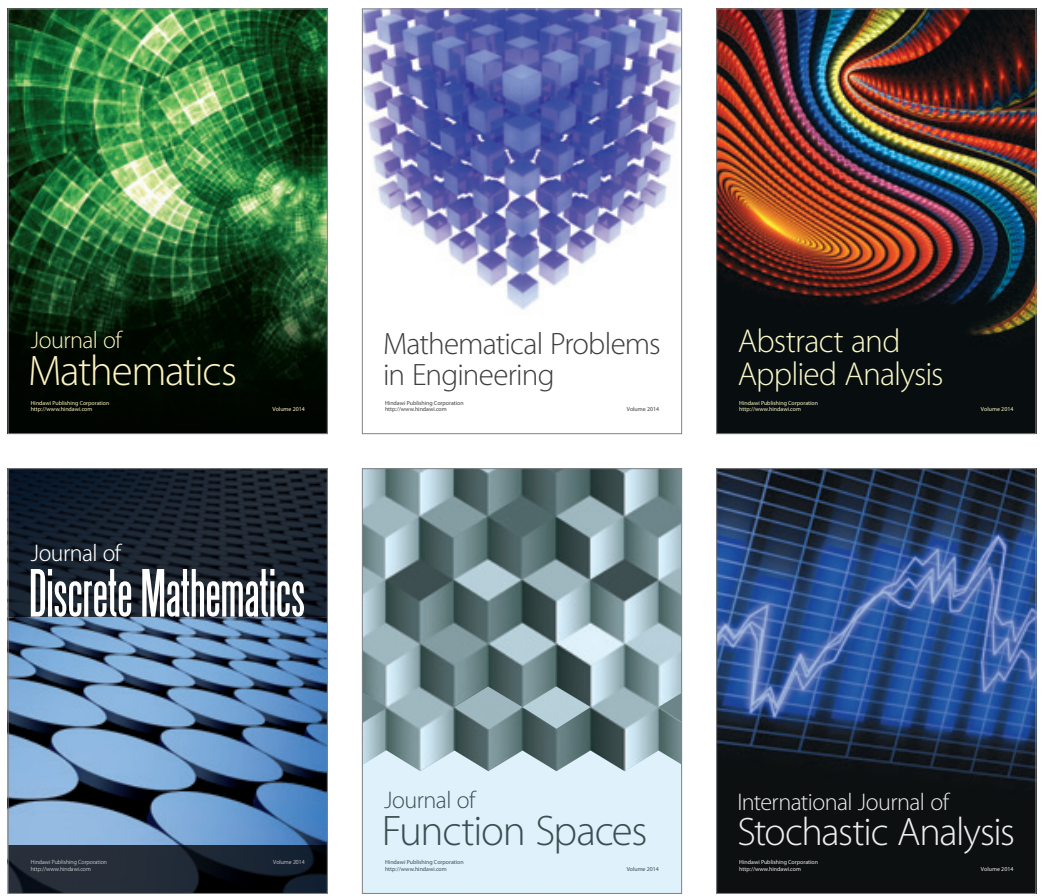

Journal of

Function Spaces

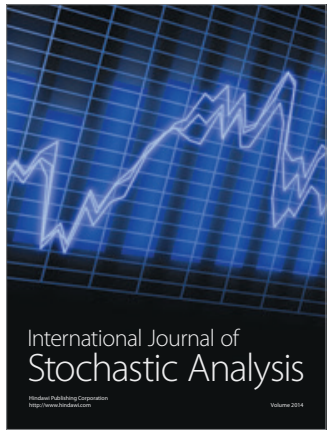

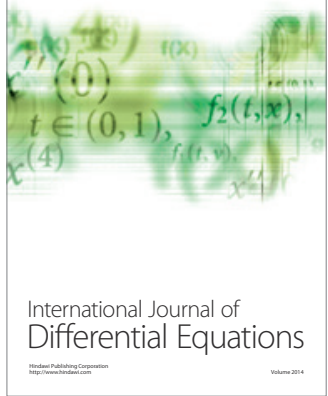
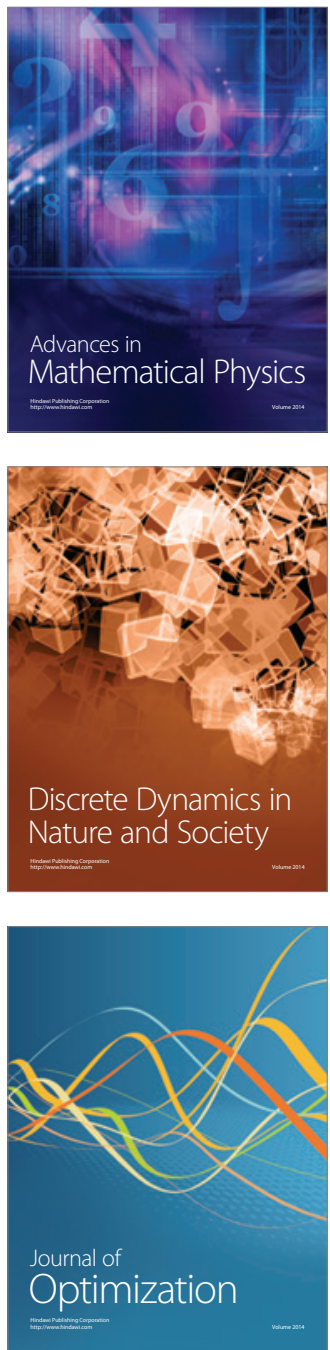